\title{
Valores organizacionales como soporte de la responsabilidad social en universidades privadas. Alianza de saberes y diálogo con el entorno
}

\author{
Yhoana Paredes Abreu \\ Cristina Seijo Suárez \\ Universidad Rafael Belloso Chacín, Maracaibo, Venezuela \\ Correo electrónico del autor: yhoana.paredes@gmail.com \\ Recepción: 19 Octubre 2017 \\ Aprobación: 15 Diciembre 2017
}

\section{Resumen}

El presente estudio tiene como propósito analizar los valores organizacionales como soporte de la responsabilidad social en universidades privadas: alianza de saberes y diálogo con el entorno, sustentado en las teorías de valores organizacionales según Seijo y Avila (2009), García y Dolan (2003), Cortina (2005) Bllanchard y O’ Connor (2005), Whetten y Cameron (2011); y para la variable responsabilidad social en Guédez (2006), De la Cuesta (2004) Fernández (2005), Pelekais, Ferrer, Cruz y Romero (2007), Pelekais y Aguirre (2008). La investigación es de tipo documental, bajo el enfoque hermenéutico, a partir de la contrastación de teorías de los diversos autores que sustentan la investigación. Las reflexiones finales sugieren las universidades privadas como institución académica pero al mismo tiempo como una organización dentro de un entorno social, deben ir más allá de las prácticas sociales promovidas desde los departamentos de extensión.

Palabras Clave: Valores organizacionales, responsabilidad social, universidades privadas, entorno. 


\title{
Organizational values such as support of social responsibility in private universities. Alliance of knowledge and dialogue with the environment
}

\begin{abstract}
The present study aims to analyze organizational values as a support for social responsibility in private universities: an alliance of knowledge and dialogue with the environment, based on theories of organizational values according to Seijo and Avila (2009), Garcia and Dolan (2003), Cortina (2005) Bllanchard and O'Connor (2005), Whetten and Cameron (2011); and for the variable social responsibility in Guédez (2006), De la Cuesta (2004) Fernández (2005), Pelekais, Ferrer, Cruz and Romero (2007), Pelekais and Aguirre (2008). The research is documentary, under the hermeneutic approach, based on the contrasting theories of the various authors that support the research. The final reflections suggest private universities as an academic institution but at the same time as an organization within a social environment must go beyond the social practices promoted from the extension departments.
\end{abstract}

Keywords: Organizational values, social responsibility, private universities, environment.

\section{Introducción}

En los últimos años, la opinión pública a nivel mundial se ha venido sensibilizando con respecto a la conducta moral que deben asumir las empresas y organizaciones, en relación a las diversas consideraciones éticas de carácter ambiental y la responsabilidad social de las mismas; pues se trata de buscar el fomento y desarrollo de un código ético basado en valores socialmente aceptables.

Sibien escierto, losvalores forman parte de la cultura organizacional y plantean límites dentro de los cuales se debe enmarcar la conducta de los individuos, bien sea en el plano organizacional como en lo personal. Por consiguiente, estos identifican y refuerzan la cultura organizacional a través del comportamiento grupal, del cual depende de la vivencia, convivencia y el logro de los objetivos de la organización. En este orden de ideas, se conceptualiza el término valores socialmente aceptables a los valores positivos como la justicia e igualdad en todas las cosas humanas, la utilidad, honestidad, belleza, salud y por el contrario los valores negativos serían sus opuestos como la injusticia, desigualdad, inutilidad, enfermedad.

En relación a esto Ferrer (2001), señala que los valores dentro de las organizaciones juegan un papel fundamental en la formación de normas o reglas del juego, pero que también dicen lo que es ético, bueno, válido, competitivo, adecuado, deseable y se van generando y 
reforzando a lo largo de la vida. Es por ello que, el talento humano no solo debe conocer la misión, visión, valores, normas, reglas de sus organizaciones sino que también, deben estar estrechamente vinculados e identificados con los mismos.

Ciertamente, los valores son cambiantes al paso del tiempo, pues si se vuelve la mirada hace algunas décadas atrás se puede apreciar que el respeto por el ambiente, entorno cultural, derecho por los consumidores, hasta el derecho de los animales no eran valores significativos como lo son en la actualidad.

Es por ello, que hoy en día se habla con cierta frecuencia de la necesidad de los cambios de valores para aminorar la problemática social, sobre todo las referidas a las de carácter ambiental y social en general, quizá esos cambios justifican el papel que juega la Responsabilidad Social.

Según refiere De la Cuesta (2004), las organizaciones desde el punto de vista moral desempeñan un papel importante dentro de la sociedad y son vistas como un ente con responsabilidad propia, bien sea económica, social y ambiental, de allí que la sociedad misma le exija comportamientos acordes con dicha responsabilidad.
En este sentido, la responsabilidad social puede entenderse como el conjunto de compromisos y obligaciones, derivado de los impactos de la gestión de las organizaciones, no solo en beneficio propio, sino también en beneficio de sus trabajadores, familias, comunidades y demás grupos de interés.

\section{Valores: cimiento de las organizaciones basado en aprendizajes estratégicos}

La palabra valor en el castellano y en otras lenguas románticas, tiene tres acepciones diferentes pero complementarias, las cuales según García y Dolan (2003), exponen en tres dimensiones, la primera ético estratégica, la cual se refiere a los valores como aprendizajes estratégicos relativamente estables en el tiempo; basados en el abordaje que una forma es mejor que otra; la segunda es la económica que es el alcance o significancia de una cosa, la tercera y última la psicológica está referida a la valentía o a la ausencia de miedo y temores para alcanzar las metas.

Según Cortina (2005), los valores son cualidades que cualifican a las personas, acciones, situaciones, sistemas y cosas; en este sentido aclara que el valor no es un objeto, cosa, o persona, sino que está en la cosa, persona, 
sociedad y en las acciones.

De la misma manera, Cortina (2005) refiere que los valores se presentan como positivos o negativos, y con dinamismo, bajo esta perspectiva considera que desde el mismo momento que se percibe un valor se capta al mismo tiempo si es positivo o negativo, en virtud que en el primer caso agrada y por ello atrae, en el segundo desagrada y por eso repele, es por ello que la autora considera que los valores positivos incitan a las personas alcanzarlos mientras que los negativos los erradica.

En el mismo sentido, Whetten y Cameron (2011) consideran que los valores forman parte de las características estables y duraderas que prevalecen en los individuos, cimientan el comportamiento y las actitudes, hasta las preferencias personales; de esta manera resultan como esa base fundamental para la toma de decisiones, lineamientos de vida y gustos personales, definiendo el comportamiento y la moralidad a lo largo de la vida.

Por su parte, Kinicki y Kreither (2003), definen los valores como las creencias perdurables en un modo de conducta y la personalidad, influyendo en el comportamiento por ser estos la guía de los actos.
Ahora bien, los valores en las organizaciones, son expresados y definidos por cada organización prácticamente desde su constitución, además constituyen los cimientos de actuación de la organización como ente social, delinean las reglas y normas de comportamiento de la organización, cuyo objetivo es el bienestar de todos los miembros.

Complementan, Whetten y Cameron (2011), que el sistema de valores en las organizaciones, se conoce como cultura organizacional, los cuales además deben ser congruentes con los de los empleados, por cuanto se sentirán productivos y satisfechos.

Sin embargo, Blanchard y O'Connor (2005), refieren que los valores no conforman una simple elección filosófica, si no que además, son necesarios para la vivencia de las organizaciones.

Así como los valores guían el comportamiento, actos y decisiones en las personas, las organizaciones no están ajenas a esa realidad, serán los valores adoptados los que influyen en la toma de decisiones y la conducta de la misma.

\section{Tipos de valores: estándares deseables de comportamiento o métodos}


El sistema de valores, tanto en las personas como en las organizaciones, orientan el comportamiento de las mismas; según Rokeach (1973), citado por Whetten y Cameron (2011), identificó dos tipos generales de valores, no relacionados entre sí, uno denominado instrumental $\mathrm{u}$ orientado a los medios, $\mathrm{y}$ el otro tipo es el terminal u orientado a los fines. El primero, plantea estándares deseables de comportamiento o métodos para lograr un fin; y el segundo, establece fines o metas deseables para el individuo.

Los valores instrumentales, se relacionan con la moralidad y la competencia, para Whetten y Cameron (2011) son los que plantean estándares deseables de comportamiento o el método para lograr un fin.

De igual manera, los valores terminales establecen fines o metas deseables para el individuo y son estos personales o morales. Es pertinente resaltar, que es necesaria la congruencia entre los valores de los empleados con los establecidos por la organización, pero se debe dar prioridad a los valores sociales, y de igual manera disminuir los valores personales $\mathrm{u}$ organizacionales que solo brindan beneficios en el comportamiento individual.

\section{Responsabilidad social: origen de}

\section{nuestros actos y consecuencias}

Antes de comenzar a estudiar el tema de la responsabilidad social es importante conocer la etimología de la palabra, de acuerdo al Diccionario de Términos Éticos (1999), citado por Pelekais, Cruz, Ferrer y Romero (2007, p.27) "la responsabilidad viene del latín responsum (respuesta) y dare (dar), que significa responder de lo hecho, nuestros propios actos y sus consecuencias, bien ante uno mismo, por la conciencia, o ante alguien"; en este sentido se interpreta entonces que ser responsable es dar respuesta a todos los implicados, acorde a los y acciones derivados de los mismos.

Ahora bien, hablar de responsabilidad social no es algo tan novedoso como parece, aun cuando en estos últimos años está adquiriendo una nueva dimensión debido a los cambios de paradigma organizacional y exigencias de la realidad contemporánea.

De manera específica, según Fernández (2005), la responsabilidad social puede definirse como la obligación asumida por las empresas de reconocer sus actos ante la sociedad, lo que conlleva a maximizar sus efectos positivos sobre la sociedad y al mismo tiempo, minimizar sus 
efectos negativos, lo que permitirá gozar de una posición más sólida respecto a su competencia, resulta ventajoso y competitivo, establecer de forma clara su pertinencia con el entorno.

Por su parte, Carroll (1999), expresa que la responsabilidad social empresarial forma parte esencial de la lengua empresarial, la cual ha ido evolucionando no solo en la teoría, si no también en lo práctico, pues significa ser congruente con lo que espera la sociedad de interés de la comunidad empresarial; de igual manera supone que la responsabilidad social tendrá nuevas definiciones, que recogerá nuevas preocupaciones tan importantes como las ya adoptadas, entre la sociedad de interés y la empresa.

Elhechodelaprácticadelaresponsabilidad social empresarial o corporativa, está referido al compromiso que la organización tiene con su entorno inmediato, sea este un ente social, o una organización económica particular; su actividad debe estar al servicio de la comunidad en la que se erige y desarrolla, además de reconocer dentro de su filosofía de gestión aquellos valores que le resulten como propios a esa sociedad.

Son entonces los valores organizacionales, la brújula que guían las acciones de las organizaciones desde el ámbito cultural, social, económico y ambiental, de allí que su desempeño impacte significativamente la sociedad, brindando bienestar social o mera rentabilidad económica.

\section{Las organizaciones y sus grupos de}

\section{interés: diálogo con el entorno}

En las últimas décadas, se viene hablando del papel fundamental que deben desempeñar las organizaciones, empresas, ONG dentro de cualquier sociedad, sean estas de carácter público, privado, nacional, internacional; se trata de la adopción de valores socialmente aceptables, de tener un comportamiento ético y respetuoso con a la sociedad y ambiente.

Dentro de las organizaciones, se identifican unos grupos de interés que afectan o son afectadas por las acciones de las mismas. Para Guédez (2006), los grupos de interés resultan todo aquello que es impactado por las acciones y decisiones de la empresa, a los cuales denomina como los relacionados, implicados, dolientes, actores o según la expresión inglesa, stakeholders.

Agrega De la Cuesta y Valor (2003), que los stakeholders son conocidos como los grupos o individuos, que afectan o son afectados por las actividades derivadas de la consecución de los objetivos de la compañía. 
Por su parte Guédez (2006), clasifica los stakeholders o grupos de interés en internos o externos, los internos tienen una relación directa con los aspectos organizacionales de la empresa y los externos, tienen una vinculación con la periferia o resonancia de las mismas, pero que se hace necesario que todos los grupos estén relacionados.

Del mismo modo, de la Cuesta y Valor (2003), clasifican en primarios y secundarios, en los cuales los primeros son los que tienen derechos directos y establecidos entre ellos identifican a los accionistas, empleados, consumidores, proveedores, gobierno; los segundos, sus derechos sobre los recursos de la empresa están establecidos en la ley o se basan en criterios como la lealtad a las comunidades, entre ellos la sociedad en general.

De igual manera, Fernández (2005), fundamentado en el libro verde presenta la división de los grupos de interés o stakeholders en dos dimensiones, a saber: una interna que hace referencia a las prácticas responsables de la empresa en lo relativo a la relación con los trabajadores y el ambiente, concretamente se abordan los siguientes puntos:

a) Gestión de los recursos humanos.
En este indicador, se prevé que las organizaciones asuman políticas responsables que promuevan la igualdad y eviten la discriminación, además brindar capacitación y fortalecer el aprendizaje, para mantener trabajadores altamente capacitados.

b) Salud y seguridad en el lugar del trabajo. Para este indicador, es necesario que las organizaciones respeten normas y criterios preventivos en materia de salud y seguridad, para que los trabajadores realicen su jornada laboral en un espacio seguro y sano.

c) Adaptación al cambio. En este caso, tanto la organización como sus trabajadores basados en un diálogo, deben estar atentos a todas las normas, recomendaciones o nuevas decisiones referidas al contexto y entorno.

d) Gestión del impacto ambiental y de los recursos naturales. Este indicador, prevé la importancia que debe tener una adecuada gestión ambiental, se trata de promover los valores ecologistas y despertar la conciencia de todos los trabajadores, reduciendo el impacto ambiental que esta 
pueda tener.

Ahora bien, en el contexto externo la responsabilidad social aborda su entorno inmediato, a saber:

a) Comunidades locales. La responsabilidad social persigue la integración de las organizaciones y empresas con su entorno inmediato como lo son las comunidades donde se establecen, por cuanto, su prosperidad depende también de la aceptación y apoyo de la comunidad.

b) Socios, comerciales, proveedores y consumidores. En este apartado se trata de ser competitivos en el mercado, esencialmente brindar una fuerte ventaja en costos y calidad. Es decir ofrecer, a todos ellos los servicios, productos que necesitan y desean.

c) Derechos humanos. Este indicador, alude a la importancia de los derechos humanos sobre los derechos laborales, los primeros cimientan la base de los segundos, es decir, se debe tener clara la importancia del ser humano como persona, que como un simple trabajador que cumple una función determinada, por ello se requiere la participación de entes como autoridades públicas, sindicatos, otros.

d) Problemas ecológicos mundiales. Dado el impacto que tienen las diversas actividades de todas las organizaciones deben establecer objetivos o metas que reduzcan el impacto ambiental.

Las Universidades Privadas, como ente social están llamadas a reflexionar sobre las problemáticas de la sociedad, con especial interés las de su entorno local, es decir, con sus grupos de interés, sean estos internos o externos; aun cuando se sabe que las Universidades sea su naturaleza, pública o privada, no impactan de manera negativa a la sociedad, todo lo contrario, solo que deberían concentrar mayores esfuerzos, en investigaciones que brinden soluciones reales a los problemas estudiados. Se encuentra entonces, que las universidades a través de los departamentos o direcciones de extensión, mantienen una alianza con las comunidades locales.

De acuerdo a lo expresado por Seijo y Ávila (2009)

...las organizaciones pueden jugar un papel importante en la creación de nuevos valores en la gente. Para esto, los líderes 
pueden apoyarse de muchas herramientas, considerando que las más importantes son: la creación de una visión motivadora, saber escuchar a la gente, la comunicación efectiva, educarlas con el ejemplo, promover formas de comportamiento que, con su reiteración, se conviertan en hábitos. (p.33).

A partir de esta reflexión, y de una manera más amplia se devela el rol transformador que se puede ejercer desde las Universidades, sobre todo la comunicación efectiva y un diálogo sincero entre los grupos de interés. Se trata pues, de mantener una estrecha relación de manera sustentable, que permita el fortalecimiento de sus grupos de interés no solo mediante las potencialidades propias de la universidad, es decir, uso exclusivo de sus fortalezas sino también que pueda satisfacer de manera positiva, sus necesidades o demanda.

\section{Componentes de la responsabilidad} social: respuesta a las demandas sociales

La responsabilidad social como respuesta a las demandas sociales se puede distinguir según Carroll (1979), citado por Fernández (2005), cuatro componentes a saber: económica, legal, ética y filantrópica.

Según Rodríguez, Fernández y Martin-Castilla (2007), desde una perspectiva multidimensional, la responsabilidad social tiene que afrontar en su totalidad todo el espectro de las obligaciones que debe la empresa hacia la sociedad, como lo son la obtención de beneficios, obedecer la ley, ser ético y ejercer ciudadanía corporativa.

En este mismo sentido, se adquieren los compromisos éticos, legales, económicos y filantrópicos, los cuales tienen la misma valía y por ende deben cumplirse simultáneamente. La responsabilidad económica va más allá de la maximización de los beneficios derivados de su actividad, es cierto que las organizaciones por su naturaleza se crean para proporcionar unos bienes y servicios, pero su responsabilidad recae en la satisfacción de las necesidades de sus consumidores a cambio de un precio justo.

En este sentido, la responsabilidad económica es demandada por la sociedad, de la misma manera, la responsabilidad legal se exige de manera vinculante y es por ello que se espera y se exige que las empresas actúen conforma a la ley, las normas que rigen tanto el mercado como la sociedad de la cual forman parte.

En cuanto a su responsabilidad ética, es esperada por la sociedad ante las acciones ejercidas; por ello Pelekais y Aguirre (2008) señalan que este tipo de responsabilidad abarcan aspectos diferentes como el ambiente o la 
responsabilidad ambiental, la contribución al desarrollo de los pueblos, justicia social, respeto a los derechos humanos, respeto hacia los consumidores; no se trata simplemente de dar respuesta a las acciones ejercidas sino también actuar conforme al marco legal y normas de la sociedad en la que se desarrolla.

Por último, está el componente de la responsabilidad filantrópica, que no es más que una acción esperada por la sociedad, en aras del bienestar de todos, es decir, la sociedad espera que las empresas actúen como un buen ciudadano, que sus acciones se circunscriban más allá del interés económico y técnico de la empresa.

\section{Consideraciones finales}

Los valores como brújula del comportamiento, guían actos y decisiones en las personas; las organizaciones no están ajenas a esa realidad, por ello los valores adoptados por las mismas definen su accionar dentro y fuera de la organización. De allí pues, que las organizaciones asuman de acuerdo a su naturaleza, el impacto social y la responsabilidad con su entorno.

En lo que refiere a las universidades privadas, como ente social generador de conocimiento, están llamadas a sumar esfuerzos para aminorar la problemática social, desde la formación integral y sensibilización socioambiental de sus estudiantes, desarrollo de investigaciones que brinden soluciones a problemas concretos de sus grupos de interés, erradicando de esta manera, la cultura reproductiva del conocimiento como legitimación del claustro universitario. Las universidades por su rol transformador, deben ir más allá, siempre vanguardistas, enseñando con el ejemplo, abierta a las necesidades propias de la sociedad, a través de la participación y el diálogo sincero de todos sus grupos de interés.

Al respecto, Borrella, Lemonche, Martínez,.Mataix, Sánchez y Suárez (2011), refieren que el diálogo con los grupos de interés supone, la creación de espacios de comunicación, para conocer los impactos de la empresa, se trata de ir más allá de una simple donación económica o material, es convenir un mayor compromiso a largo plazo.

\section{Referencias bibliográficas}

Blanchard, K.; O'Connor, M. (2005). Administración por valores. Editorial Norma, S.A. Bogotá - Colombia.

Borrella, I.; Lemonche, P.; Martínez, R.; Mataix, C.; Sánchez, E.; Suarez, M. (2011). Guía de Responsabilidad Social Corporativa para Pymes. ONGAWA, Ingeniería para el Desarrollo Humano. España.

Carroll, A. (1999). Corporate Social 
Responsibility. Evolution of a Definitional Construct. En BUSINESS \& SOCIETY, Vol. 38 No. 3, September 1999, pp. 268295. Estados Unidos.

Cortina, A. (2005). El Mundo de los valores. "Ética mínima" y educación. Editorial Búho. Colombia.

De la Cuesta, M.; Valor, C. (2003). Responsabilidad social de la empresa. Concepto, medición y desarrollo en España. En boletín económico de ICE N 2755, del 20 al 26 de enero de 2003, pp 7-22. España.

De la Cuesta, M. (2004). El porqué de la responsabilidad social corporativa. En boletín económico de ICE $N$ 2813, del 2 al 5 de septiembre de 2004, pp. 46-58. España.

Fernández, R. (2005). Administración de la responsabilidad social corporativa. Thomson. España.

Ferrer, J. (2001). Presencia del componente ético en sectores de actividad industrial. Maracaibo - Venezuela.

García, S. \& Dolan, S. (2003). La dirección por valores. El cambio más allá de los objetivos. Editorial McGraw - Hill. España

Guédez, V. (2006). Ética y práctica de la responsabilidad social empresarial. Editorial Planeta. Venezuela

Kinicki, A.; Kreiter, R. (2003). Comportamiento Organizacional. McGraw-Hill. México.

Pelekais, C.; Aguirre, R. (2008). Hacia una cultura de responsabilidad social. Pearson Educación. México.

Pelekais, C.; Ferrer, J.; Cruz, A.; Romero, D. (2007). Responsabilidad social. Compromiso de las organizaciones. Contraloría del Estado Zulia. Maracaibo - Venezuela
Rodríguez, O.; Fernández, J.; Martín-Castilla, J. (2007). Percepción directiva de las relaciones entre responsabilidad social y ética empresarial. En boletín económico de ICE N 2927, del 1 al 15 de diciembre de 2007, pp. 43-56. España.

Seijo, C.; Ávila, M. (2009). Valores éticos y responsabilidad social empresarial: una plataforma gerencial en universidades privadas. Redhecs, 6, 26-40.

Whetten, D.; Cameron, K. (2011). Desarrollo de habilidades directivas. México: Editorial Pearson. 